\title{
FACTORING THE HILBERT CUBE
}

\author{
BY JAMES E. WEST
}

Communicated by Richard Anderson, June 2, 1969

In 1964, R. D. Anderson announced [1] that the product of a countably infinite collection of dendra is a Hilbert cube (a dendron being a nondegenerate, uniquely arcwise connected, Peano continuum). He and R. H. Bing conjectured in [4] that a countably infinite product of compact contractible complexes is a Hilbert cube. The purpose of this note is to announce the following theorems which affirm the above Anderson-Bing conjecture. The author wishes to thank R. D. Anderson for several helpful conversations on the subject dating as far back as 1966.

THEOREM 1. A countably infinite product of nondegenerate spaces is a Hilbert cube if the product of each space with the Hilbert cube is a Hilbert cube.

Theorem 2. If two finite simplicial complexes have the same simple homotopy type, then their products with the Hilbert cube are homeomorphic.

For simply connected (finite) simplicial complexes, the concept of simple homotopy type coincides with that of homotopy type, since the algebraic invariant which distinguishes the two vanishes [10]. Thus, a contractible (finite) simplicial complex has the simple homotopy type of a point. Combining this with the above two theorems yields

Corollary 1. A countably infinite product of nondegenerate, contractible, finite simplicial complexes is a Hilbert cube. ${ }^{1}$

COROLlary 2. The product of a locally finite simplicial complex with the Hilbert cube is locally homeomorphic to the Hilbert cube.

This is by Theorem 2 since each point of a locally finite simplicial complex has a neighborhood which is a collapsible complex.

The next is the converse of a theorem of D. W. Henderson, who proved in [7] that each open subset of a separable, infinite-dimensional Hilbert space is homeomorphic to the product of that space with a countable, locally finite simplicial complex. Corollary 3 ap-

1 The author understands by letter that Andrej Szankowski, a student of Pelczynski, has also obtained this result. 
peared as Conjecture 2 in [6] and as such is attributed to R. D. Anderson.

COROLlaRy 3. The product of a countable, locally finite simplicial complex with a separable, infinite-dimensional Hilbert space is homeomorphic to an open subset of that Hilbert space.

Proof. Henderson proved in [8] that all separable, metrizable manifolds modelled on an infinite-dimensional Hilbert space are homeomorphic to open subsets of that Hilbert space. Bessaga and Klee [5] and Anderson [2] proved independently that the product of the Hilbert cube with the countable infinite product of lines is again the countable product of lines. Anderson also showed in [3] that separable infinite-dimensional Hilbert spaces are all homeomorphic to the countable product of lines. Putting all these results together with Corollary 2 gives Corollary 3.

Finally, because recent work of Kuiper-Burghelea [9] and Moulis [11] has shown that homotopy-equivalent open subsets of separable, infinite-dimensional Hilbert spaces are $C^{\infty}$-diffeomorphic, we have

Corollary 4. Let $M$ and $N$ be two countable, locally finite simplicial complexes, and let $H$ be an infinite-dimensional Hilbert space. Then $M \times H$ is homeomorphic to $N \times H$ if $M$ and $N$ have the same homotopy type.

Concerning PRoOfs of Theorems one AND two. Theorem 1 is a stronger version, for the setting of the Hilbert cube, of a theorem announced in [1] by Anderson. He defined the term strongly homeomorphic as follows: Let $X, Y$, and $Z$ be compact metric spaces, and say that $X \times Z$ is strongly homeomorphic to $Y \times Z$ provided that for each positive number $\epsilon$ there is a homeomorphism $g$ of $X \times Z$ onto $X \times Z$ and a map $f$ of $X$ onto $Y$ such that for each $x$ in $X$ and $z$ in $Z$, the distance between the $Y$-coordinate of $g(x, z)$ and $f(x)$ is less than $\epsilon$. He then proved that if $\left\{X_{i}\right\}_{i=1}^{\infty}$ and $\left\{Y_{i}\right\}_{i=1}^{\infty}$ are sequences of compact metric spaces such that for each $i>0, Y_{i}$ is homeomorphic to $Y_{i+1}$ and $X_{i} \times \prod_{j>i} Y_{j}$ is strongly homeomorphic to $Y_{i} \times \prod_{j>i} Y_{j}$, then $\prod_{i>0} X_{i}$ is homeomorphic to $\prod_{i>0} Y_{i}$. The author first obtains a proof of this theorem without the full strength of the definition of strongly homeomorphic, requiring only that for each $\epsilon>0$, there be a homeomorphism $g$ of $X \times Z$ onto $Y \times Z$ such that for all $x$ in $X$, the diameter of the image of the projection into $Y$ of $g(\{x\} \times Z)$ is less than $\epsilon$.

Letting $Q$ denote the Hilbert cube and $I$ the unit interval, the next step in the proof of Theorem 1 is to show that if $\left\{Y_{i}\right\}_{i=1}^{\infty}$ is any collec- 
tion of nondegenerate spaces such that for each $i, Y_{i} \times Q$ is homeomorphic to $Q$, then $\prod_{i>0} Y_{i}$ has the property that for each $\epsilon>0$ there is a homeomorphism $g$ of $\left(\prod_{i>0} Y_{i}\right) \times Q$ onto $I \times Q$ such that the diameter of the projection into $I$ of $g(\{y\} \times Q)$ is less than $\epsilon$ for all $y$ in $\prod_{i>0} Y_{i}$.

Finally, any countably infinite collection $\left\{X_{i}\right\}_{i=1}^{\infty}$ of nondegenerate spaces, each of whose product with the Hilbert cube is a Hilbert cube, may be written as an infinite union of pairwise disjoint infinite collections $\left\{Y_{i, j}\right\}_{j=1}^{\infty}$ of such spaces. Since then $\prod_{i>0} X_{i}$ is homeomorphic to $\prod_{i>0}\left(\prod_{j>0} Y_{i, j}\right)$, the previous two results combine to give that it is homeomorphic to the Hilbert cube.

The proof of Theorem 2 is longer and proceeds from the two next theorems. Let us agree to say that a compact metric space $X$ admits an interior approximation by $Y$ provided that there exists in $X$ a sequence $\left\{Y_{i}\right\}_{i=1}^{\infty}$ of homeomorphic copies of $Y$ such that $\lim \inf \left\{Y_{i}\right\}_{i=1}^{\infty}=X$ and a collection of homeomorphisms as follows:

1. there is an embedding $\alpha$ of $X$ in the Hilbert cube such that for every positive number $\epsilon$ and positive integer $i$, there is

2. an embedding $\beta_{i}^{\epsilon}$ of $X$ in the Hilbert cube with $d\left(\beta_{i}^{\epsilon}(x), \alpha(x)\right)$ $\leqq 2^{-i}$ for all $x$ in $X$ ( $d$ being a metric for the Hilbert cube) such that for each positive integer $j$ there is

3. a homeomorphism $\gamma_{i, j}^{\epsilon}$ of $\alpha(Y)$ onto $\beta_{i}^{\epsilon}\left(Y_{i+j}\right)$ with $d\left(\gamma_{i, j}^{\epsilon}(x), x\right)$ $\leqq \epsilon$ for all $x$ in $\alpha\left(Y_{i}\right)$.

With this definition, one may prove

TheOREM 3. If $X$ admits an interior approximation by $Y$, then $X$ and $Y$ are homeomorphic.

A further definition is the following: Let $X$ and $Y$ be compact metric spaces. A closed subset $Z$ of $X \times Y$ will be called a $Y$-approximation to $X$ provided that for each $\epsilon>0$ there is an embedding $g_{\mathrm{e}}$ of $X \times Y$ in $Z \times Q \subset X \times Y \times Q$ such that $g_{\mathrm{e}}$ changes the $X$-coordinate of no point more than $\epsilon$ and the following condition is satisfied: Let $\bar{g}_{\epsilon}:(X \times Y) \times Y \rightarrow(Z \times Q) \times Y$ be the embedding obtained from $g_{\epsilon}$ by taking the product of $g_{\mathrm{e}}$ with the identity map in the new $Y$-coordinate. Also, define $\bar{Z}=\left\{\left((x, y), y^{\prime}\right) \in(X \times Y) \times Y \mid\left(x, y^{\prime}\right) \in Z\right\}$. The additional condition to be satisfied is that there be a homeomorphism $h_{\epsilon}$ from $\bar{g}_{\epsilon}(\bar{Z})$ onto $Z \times Y$ which changes no point's $(X \times Y)$-coordinate more than $\epsilon$.

From Theorem 3, one may prove

THEOREM 4. If $Z$ is a $Y$-approximation to $X$, then $Z \times \prod_{i>0} Y_{i}$ is homeomorphic to $X \times \prod_{i>0} Y_{i}$ (where $\left\{Y_{i}\right\}_{i=1}^{\infty}$ is a collection of homeomorphic copies of $Y$ ). 
Theorem 2 may be proved from Theorem 4 in the following way: Whitehead [12] showed that two finite simplicial complexes $K$ and $L$ are of the same simple homotopy type if and only if there is a finite sequence $K=K_{1}, K_{2}, \cdots, K_{n}=L$ of finite simplicial complexes such that for all $i=1, \cdots, n-1$, either $K_{i+1}$ may be obtained from $K_{i}$ by an elementary simplicial collapse or $K_{i}$ may be so obtained from $K_{i+1}$. The definition of an elementary simplicial collapse is as follows: Suppose $M$ is a simplicial complex, $\Delta$ is a simplex of $M$ contained in no other, and $\Delta^{\prime}$ is a proper face of $\Delta$ properly contained in no other simplex of $M$. Then the transformation $M \rightarrow M \backslash\left(\Delta^{\circ} \cup \Delta^{\prime 0}\right)$ is an elementary simplicial collapse, where $\Delta^{\circ}$ and $\Delta^{\prime 0}$ are the open simplices. It is shown that if $K$ collapses to $L$ by an elementary simplicial collapse, then the set $(K \times\{0\}) \cup(L \times I)$ is an $I$-approximation to $K$. Hence, by Theorem $4, K \times Q$ is homeomorphic to [ $(K \times\{0\}) \cup(L \times I)]$ $\times Q$, which is then shown to be a Hilbert cube. Theorem 2 then follows immediately by induction.

The author has recently extended these techniques to obtain the following results from a generalization of Corollary 3 (coupled with some recent work of D. W. Henderson, Stable classification of infinitedimensional manifolds by homotopy type, Ann. of Math. (to appear):

(1) if $E$ is a Banach space homeomorphic to its countable infinite Cartesian power, then each paracompact E-manifold is (homeomorphic to) the product of $E$ with a locally finite-dimensional metric simplicial complex and

(2) if $E$ is a Banach space homeomorphic to its countably infinite Cartesian power, then each paracompact E-manifold has an open cover by (topological) E-balls which is locally finite, is closed under nonempty intersections, and whose nerve has the homotopy type of the manifold.

\section{REFERENCES}

1. R. D. Anderson, The Hilbert cube as a product of dendrons, Notices Amer. Math. Soc. 11 (1964), 572, Abstract 614-149.

2. - Hilbert space is homeomorphic to the countable infinite product of lines, Bull. Amer. Math. Soc. 72.(1966), 515-519.

3. - Topological properties of the Hilbert cube and the infinite product of open intervals, Trans. Amer. Math. Soc. 126. (1967), 200-216.

4. R. D. Anderson and R. H. Bing, A complete elementary proof that Hilbert space is homeomorphic to the countable infinite product of lines, Bull. Amer. Math. Soc. 74 (1968), 771-792.

5. C. Bessaga and V. L. Klee, Every non-normable Fréchet space is homeomorphic with all its closed convex bodies, Math. Ann. 163 (1966), 161-166.

6. D. W. Henderson, Infinite-dimensional manifolds, Proc. Internat. Sympos. Topology Appl., Herceg Novi, Jugoslavia, 1968.

7. - Open subsets of Hilbert space, Compositio Math. (to appear). 
8. - Infinite-dimensional manifolds are open subsets of Hilbert space topology (to appear).

9. N. H. Kuiper and D. Burghelea, Hilbert manifolds, Ann. of Math. (to appear).

10. J. Milnor, Whitehead torsion, Bull. Amer. Math. Soc. 72 (1966), 358-426.

11. N. Moulis, Sur les variêtés Hilbertiennes et les fonctions non-dégénérés, Indag. Math. 30 (1968), 497-511.

12. J. H. C. Whitehead, Simplicial spaces, nuclei, and m-groups, Proc. London Math. Soc. (2) 45 (1939), 243-327.

University of Kentucky, Lexington, Kentucky 40506 